\title{
Wing and propeller aerodynamic interaction through nonlinear lifting line theory and blade element momentum theory
}

\author{
Hospodár̆ Pavel ${ }^{{ }^{*}}$, Klesa Jan ${ }^{1}$, Žižkovský Nikola ${ }^{1}$ \\ ${ }^{1}$ Faculty of mechanical engineering, Czech technical university, Prague, Czech Republic
}

\begin{abstract}
In this paper a computational methodology of aerodynamic interaction between propeller and wing is described. Presented work is focused on development of quick and accurate tool. Lifting line theory (LLT) with nonlinear airfoil characteristic is used to solve a finite span wing aerodynamic to predict downwash and lift distribution respectively. Blade element momentum theory (BEM) is used as a computational tool for estimating total thrust, torque, axial and tangential velocity distributions. Model of slipstream development is considered. Influence of propeller model to wing is simulated as contribution of higher dynamic pressure and change of angle of attack behind the propeller.
\end{abstract}

\section{Introduction}

One of the aviation pioneer Otto Lilienthal described elementary causality in aerodynamic [1]. He was probably the first who observed that aerodynamic forces and moments depends on a value of airspeed. This can be deduced that a required lift force can be produced by moving of an airplane in sufficient freestream velocity or the airplane stays at the position and freestream is generated by propellers. Second way is a principle that is trying to benefit in distributed propeller systems.

During second half of twenty century were intensively investigated airplanes with different vertical/short take-off and landing configurations. Wind tunnel results show higher aerodynamic efficiency when propeller is mounted in the front of the wing [10], [11]. Especially in design of electrical airplanes, that enables higher modularity, an engines are often placed in the wing. Different computational approaches are used to estimate propeller wing aerodynamic interaction. To solve propeller wing interaction using CFD is performed in [12]. It is high-fidelity computation method that provides detailed description of flow field. On the other hand, it is time consuming to prepare a geometry, computational mesh and a calculation over angle of attack sweep. More suitable solution for design is described in [9]. He used vortex lattice method to solve wing's aerodynamic and propeller is simulated by blade element theory.

\footnotetext{
"Corresponding author: pavel.hospodar@fs.cvut.cz
} 
In this paper a non-linear lifting line theory is used to solve induced and viscous part of aerodynamic. Further blade element momentum theory determines induced velocity provided by propeller. Propeller wing interaction is connected through model of propeller slipstream. This will lead to accurate and quick tool to preliminary design and optimization.

\section{Lifting line theory}

An understanding of aerodynamic forces origin is associated with beginning of aviation. Wright brothers successfully first powered man controlled flight in 1903 was also based on knowledge of aerodynamic. They had no computational tool to predict aerodynamic forces. However, they developed kite and glider respectively to obtain data from experimental but they were frustrated because the lift produced by glider were only $1 / 3$ of lift which was predicted by using Lilienthal dat. They decided to make their own wind tunnel to measure of lift and drag forces.

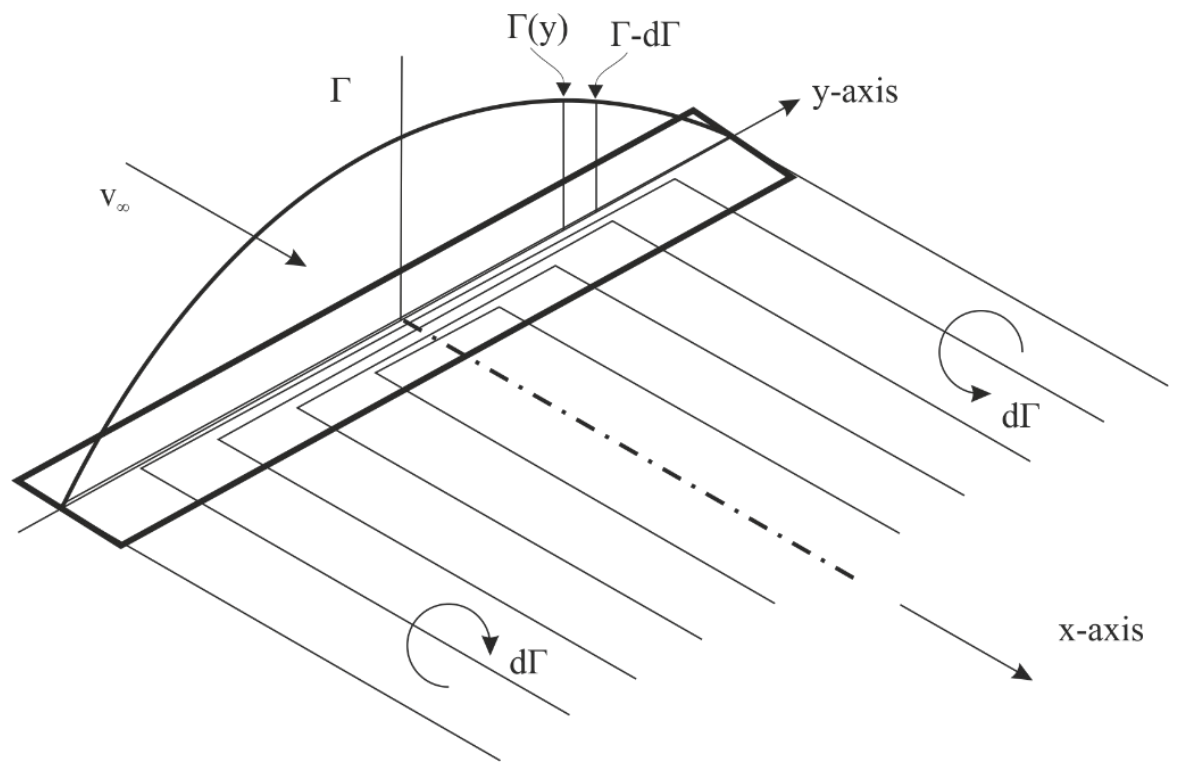

Fig. 1. Spanwise distribution of circulation

The first description of the aerodynamic forces was published as Lifting line theory [2], three years later in English translation [3]. In this paper a computation of lift and drag forces for finite span wing was described. Using this methodology an optimal lift distribution with minimum induced drag was derived - elliptical wing.

Lifting line theory [2] is based on a description of flow field using knowledge form an electromagnetic field. Specifically, it is Biot-Savart law: velocity produced by a semi-infinite segment of a vortex filament. Prandtl described a vortex scheme as a set of horseshoe vortices, the carrier parts of which lie in a single vortex line connecting the aerodynamic centres of the profile. A value of local vortex $\Gamma(y)$ equals a sum of all vortices which going through local point. Vortex distribution has a consequence on flow field airspeed distribution. Each vortex induces downwash in near of the flow field and their orientation a magnitude 
determines local induced angle of attack. Downwash $w$ in the wing side position $y_{0}$ equation (called Prandtl's equation) is defined as follows:

$$
w\left(y_{0}\right)=\frac{1}{4 \pi} \int_{-b / 2}^{b / 2} \frac{d \Gamma}{y-y_{0}}
$$

To solve previous equation, a knowledge of wing geometry and circulation distribution is necessary. However, it is directly influenced by the induced angle of attack and downwash velocity respectively. It is implicit equation and must be solved numerically. This solution of the Prandtl's equation allows for the calculation of the finite span wing with dihedral, sweep, twist and different shape of airfoil respectively. Further, it can be used a non-linear aerodynamic characteristic of the airfoil such as lift and drag forces and pitch moment coefficient and the effect of Reynolds number.

Then a computation process is described. Geometrical model of the wing, a panel distribution, is created in the first step and the initial induced angle of attack distribution is estimated (it should be zero). In the first step of iteration a total angle of attack $\alpha_{\text {tot }}$ is computed as a sum of induced angle of attack $\alpha_{i}$, geometrical angle $\alpha_{g}$ and twist angle $\alpha_{t}$ :

$$
\alpha_{t o t}=\alpha_{i}+\alpha_{g}+\alpha_{t}
$$

In the next step a local lift coefficient $c_{L}$ is interpolated from non-linear aerodynamic characteristic based on total angle of attack. Local lift force $l$ depends on air density $\rho$, air speed $V^{2}$, local lift coefficient and local chord $c$ and is computed by following terms:

$$
l=\frac{1}{2} \rho \cdot V^{2} \cdot c_{L} \cdot c
$$

Then a Kutta-Joukovsky law is used to determine local circulation:

$$
\Gamma=\frac{1}{2} V \cdot c_{L} \cdot c
$$

A computed circulation is then substituted to the Prandtl's equation (1) and new downwash is determined. If the difference between computed and entered value is higher than setting limit a procedure is repeated until the convergence criteria are satisfied. This procedure is depicted on Fig. 2. 


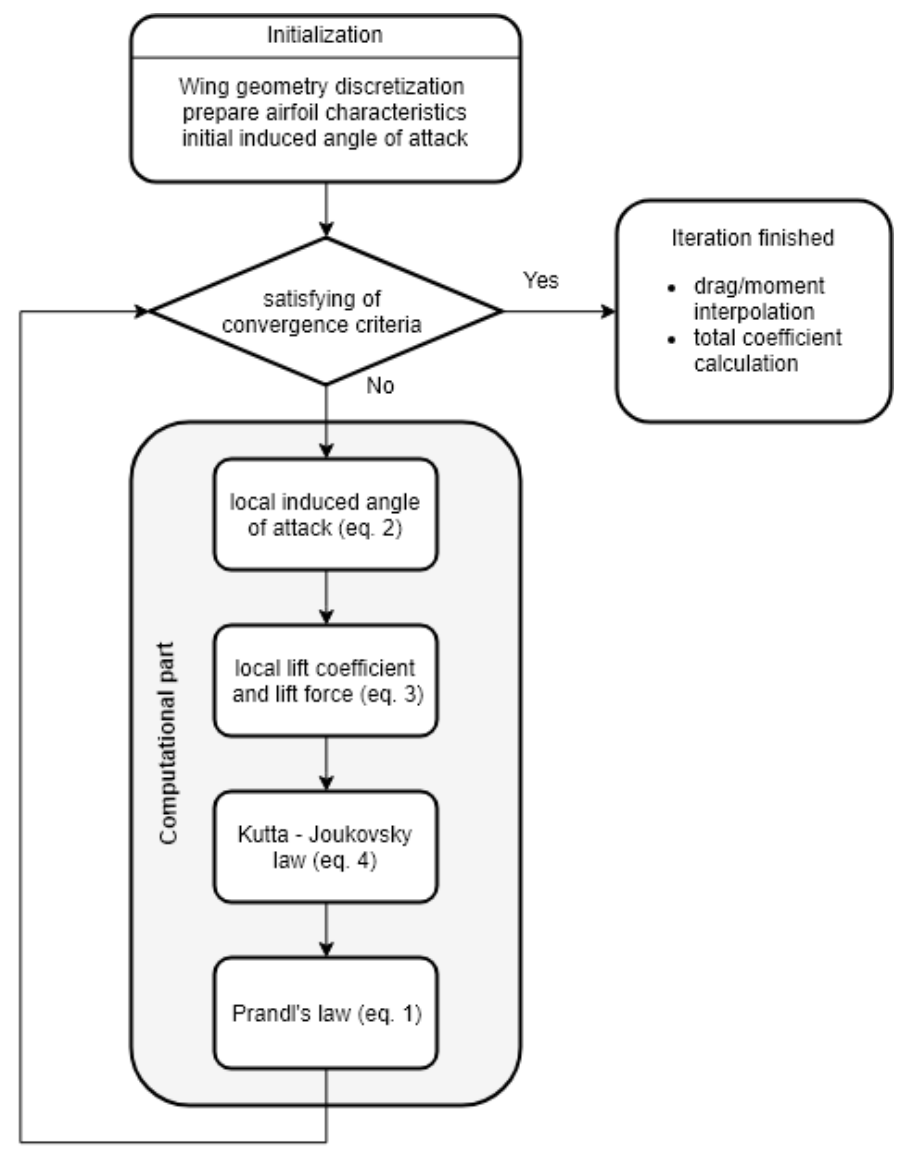

Fig. 2. LLT computational process

Note, that relation between induced angle of attack and downwash is defined in non-linear form as follows:

$$
\alpha_{i}=\tan ^{-1} \frac{w}{V}
$$

\section{Blade element momentum theory}

Combination of geometric properties of the propeller and conservation law of momentum [6], [7] a blade element momentum theory is developed. This methodology provides detailed information about flow filed and enables to design geometry of the propeller - twist and chord distribution [8]. Thrust and torque are determined as a sum of small elemental section along the blade span. Figure 3 shows the velocity and force diagram on elemental blade section. 


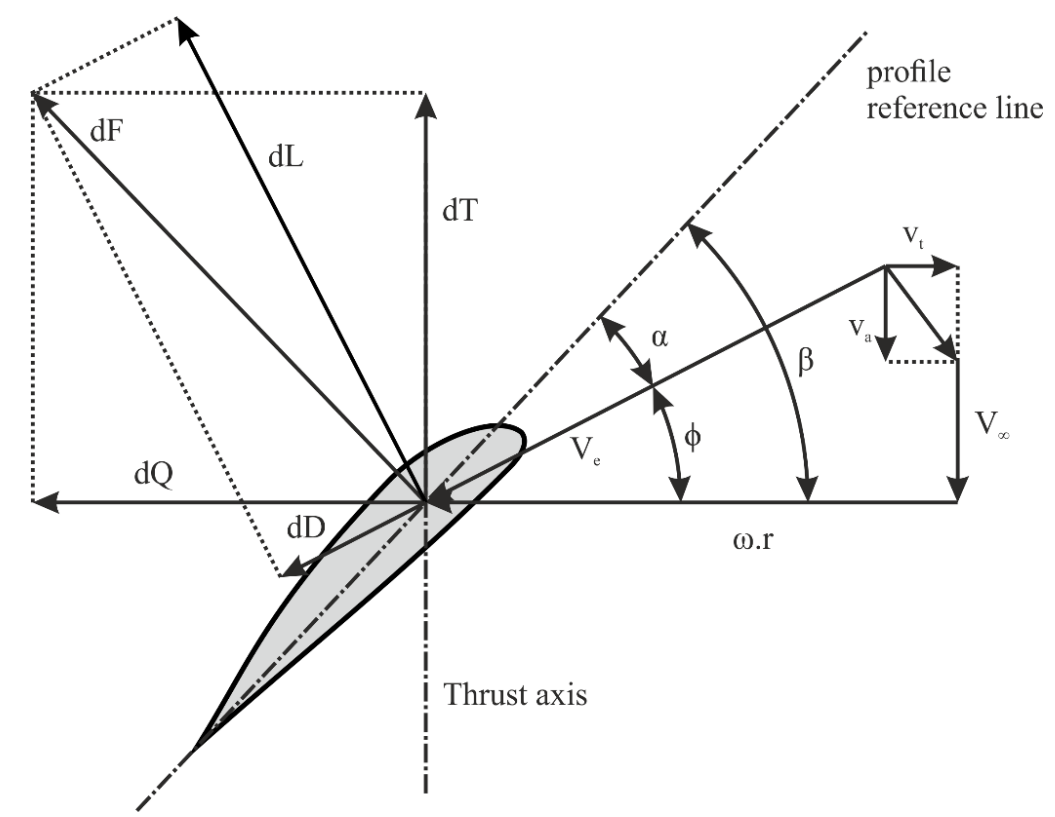

Fig. 3. Local velocity and force acting on a propeller blade element for BEM theory

A circular stream tube is supposed than axial and tangential parts of conservation law of momentum between $r$ and $r+d r$ are defined as:

$$
\begin{aligned}
& 2 \pi r d r \rho\left(V_{\infty}+v_{a}\right) v_{a}=d T \\
& 2 \pi r d r \rho\left(V_{\infty}+v_{a}\right) v_{t}=\frac{d Q}{r}
\end{aligned}
$$

Where $r$ is local radius, $d r$ is elementary area, $v_{a}$ and $v_{t}$ are induced axial and tangential velocity respectively, $d T$ is elemental thrust and $d Q$ elemental torque. Considering an aerodynamic lift $d L$ and drag $d D$ forces action on blade element, the torque and thrust can be described as:

$$
\begin{aligned}
& d T=B(d L \cos (\varphi)-d D \sin (\varphi))=B\left(c_{l} \cos (\varphi)-c_{d} \sin (\varphi)\right) \frac{1}{2} \rho V_{e}^{2} c d r \\
& \frac{d Q}{r}=B(d L \sin (\varphi)+d D \cos (\varphi))=B\left(c_{d} \sin (\varphi)+c_{d} \cos (\varphi)\right) \frac{1}{2} \rho V_{e}^{2} c d r
\end{aligned}
$$

where $B$ is number of blades and $\varphi$ is inflow angle and is defined:

$$
\varphi=\operatorname{atan} \frac{V_{\infty}+v_{a}}{\omega r-v_{t}}
$$

From figure 5 an effective velocity $V_{e}$ may be write as a function of freestream velocity $V_{\infty}$, rotational velocity $\omega r$ and induced tangential $v_{t}$ and axial $v_{a}$ velocity respectively:

$$
V_{e}=\frac{V_{\infty}+v_{a}}{\sin (\varphi)}=\frac{V_{\infty}(1+a)}{\sin (\varphi)}
$$




$$
V_{e}=\frac{\omega r-v_{t}}{\cos (\varphi)}=\frac{\omega r\left(1-a^{\prime}\right)}{\cos (\varphi)}
$$

where $V_{\infty}$ is freestream velocity and $\omega r$ is radial velocity. When radial location is close to tip of the blade a lift goes to zero due to pressure equalization. A tip loss factor [7] describes this effect as:

$$
\begin{gathered}
F=\frac{2}{\pi} \operatorname{acos}\left(e^{-f}\right) \\
f=\frac{B}{2} \frac{R-r}{r} \frac{1}{\sin (\varphi)}
\end{gathered}
$$

Hence induced velocities are multiplied by tip loss factor. Equations (11) and (12) can be substituted into equation (8) and (9) and combined with (6) and (7). Thus a final form of BEM theory with using tip loss factor is given:

$$
\begin{gathered}
\frac{a}{1+a}=\frac{\sigma}{4 F \sin ^{2}(\varphi)}\left(c_{l} \cos (\varphi)-c_{d} \sin (\varphi)\right) \\
\frac{a^{\prime}}{1-a^{\prime}}=\frac{\sigma}{4 F \sin (\varphi) \cos (\varphi)}\left(c_{d} \sin (\varphi)+c_{d} \cos (\varphi)\right)
\end{gathered}
$$

where $\sigma=B c / 2 \pi r$ is local solidity of the disk. Iterative process of BEM theory is depicted on the following figure. For the solution a simple iterative process can be used: $a(i t+1)=$ $a(i t-1)+\varepsilon(a(i t)-a(i t-1))$, where $0<\varepsilon<1$.

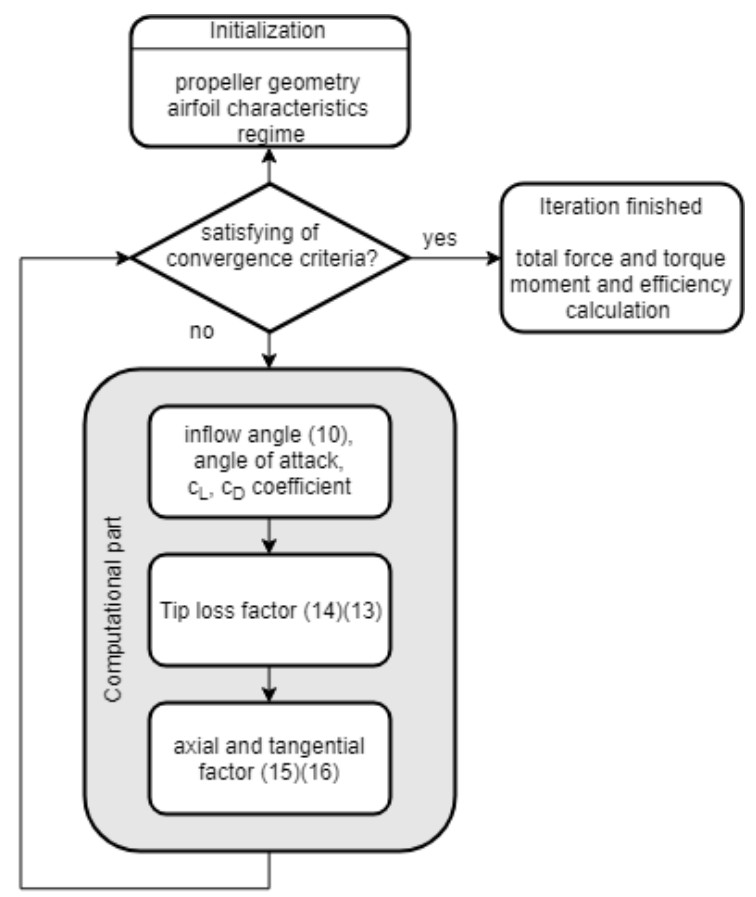

Fig 4. BEM computational process 
At the end of BEM computational process, a local thrust and torque moment is computed (8)(9) and total thrust and torque moment is integrated. Finally thrust $c_{T}$ and power $c_{p}$ coefficient, the efficiency $\eta$ and advance ratio $\lambda$ are defined:

$$
\begin{gathered}
c_{T}=\frac{T}{\rho n^{2} D^{4}} \\
c_{p}=\frac{P}{\rho n^{3} D^{5}} \\
\eta=\frac{c_{T}}{c_{p}} \lambda \\
\lambda=\frac{V_{\infty}}{n D}
\end{gathered}
$$

\section{Propeller-wing interaction}

Propeller wing aerodynamic interaction is described in this part. Basic computational solution of propeller effect on wing is to use propeller forces and add them to boundary condition of the wing. Thus, the propeller aerodynamic is computed separately at the beginning of computation and then induced velocities produced by propeller are used in LLT. Model of propeller slipstream describes velocity increasing behind the propeller disc. Induced velocities depends on position wing and propeller [4] therefore slipstream development factor is defined as follows:

$$
k_{d}=1+\frac{s}{\sqrt{s^{2}+R^{2}}}
$$

where $s$ is distance propeller disk and wing panel and $R$ is propeller radius. The value of development factor is approaches 2 if distance is infinity and 1 if $s$ is zero. It is direct consequence of momentum equations. Having the development factor an axial velocity distribution can be determined:

$$
v_{i a}^{\prime}=k_{d} v_{i a}
$$

where $v_{i a}$ is propeller induced axial velocity component. The development of the axial and tangential velocities as suggested by [9] is used. Mid chord control points of the wing are checked if is located inside the propeller area. Local wing's airspeed is than computed as follows:

$$
\begin{gathered}
v_{x}=V_{x}+v_{i a}^{\prime} \\
v_{y}=V_{y}+v_{i t}^{\prime} \sin (\psi)
\end{gathered}
$$

where $V_{x}$ is axial freestream velocity component, $V_{y}$ is vertical airspeed, $v_{i t}^{\prime}$ is tangential component of induced propeller velocity and $\psi$ is angular of the propeller blade. 


\section{Acknowledgment}

Authors acknowledge support from the EU Operational Programme Research, Development and Education, and from the Center of Advanced Aerospace Technology (CZ.02.1.01/0.0/0.0/16_019/0000826), Faculty of Mechanical Engineering, Czech Technical University in Prague.

\section{References}

1. O. Lilienthal: Der Vogelflug als Grundlage der Fliegekuns

2. L. Prandtl Tragflügeltheorie, Königliche Gesellschaft der Wissenschaften zu Göttingen, 1918

3. L. Prandtl: Applications of modern hydrodynamics to aeronautics, NACA Report No 116,1921

4. B. W. McCormick: Aerodynamics of V/STOL Flight, 1st ed., Academic Press, New York, 1969

5. L. Prandtl and A. Betz, Schraubenpropeller mit geringstem Energieverlust Goettinger Nachrichten, 1919

6. Froude: On the Elementary relation between pitch, slip and propulsive efficiency, Transactions of the Institution of Naval Architects, Vol, XIX, 1889

7. Rankine: On the Mechanical Principle of the Action of Propellers, Transactions of the Institution of Naval Architects. Vol. VI, 1865

8. M. H. McCrink and J. W. Gregory: Blade Element Momentum Modeling of LowReynolds Electric Propulsion Systems, Journal of Aircraft, Vol. 54, pp. 163-176, 2017

9. R. H. Stone: Aerodynamic Modeling of the Wing-Propeller Interaction for a Tail-Sitter Unmanned Air Vehicle, Journal of Aircraft, Vol. 45, No. 1, pp. 198-210, 2008

10. R. E. Kuhn and H. W. Draper: An investigation of a wing-propeller configuration employing large-chord plain flaps and large-diameter propellers for low-speed flight and vertical take-off, NACA Technical Note 3307, National Advisory Committee for Aeronautics. Langley Aeronautical Lab.; Langley Field, VA, United States, 1954

11. W. C. Hayes, R. E. Kuhn and I. R. Sherman: Effects of Propeller Position and Overlap on the Slipstream Deflection Characteristics of a Wing-propeller, NACA Technical Note 4404, National Advisory Committee for Aeronautics. Langley Aeronautical Lab.; Langley Field, VA, United States, 1958

12. A. Jirasek, P. Aref, M. Ghoreyshi, M. Satchell, and K. Bergeron: Computational Study of Propeller Wing Aerodynamic Interaction, Aerospace 2018, 5(3), 79, 2018 